Service social

\title{
Au-delà des soins : un travail de conciliation
}

\section{Nancy Guberman et Pierre Maheu}

Volume 43, numéro 1, 1994

Maintien à domicile

URI : https://id.erudit.org/iderudit/706644ar

DOI : https://doi.org/10.7202/706644ar

Aller au sommaire du numéro

Éditeur(s)

École de service social de l'Université Laval

ISSN

1708-1734 (numérique)

Découvrir la revue

Citer cet article

Guberman, N. \& Maheu, P. (1994). Au-delà des soins : un travail de conciliation. Service social, 43(1), 87-104. https://doi.org/10.7202/706644ar

\section{Résumé de l'article}

Cet article remet en question une vision de la prise en charge familiale des proches adultes dépendants qui réduit ce travail aux soins directs à la personne. Il montre qu'au-delà des soins s'impose un important travail de conciliation qui vise à maintenir un équilibre entre les multiples besoins et les exigences de chacune des sphères de la vie : sphères de vie personnelle, sociale et familiale; sphères liées au travail salarié et à la prise en charge. Les données qui sous-tendent cette réflexion viennent de plusieurs recherches sur le sujet menées par les auteurs depuis 1987, dont l'une porte sur la conciliation du travail salarié et des soins aux proches. Des implications pour la pratique en maintien à domicile sont dégagées en conclusion. 


\title{
Au-delà des soins : un travail de conciliation ${ }^{1}$
}

\author{
Nancy GUBERMAN \\ Professeure \\ Département de travail social \\ Université du Québec à Montréal \\ Pierre MAHEU \\ Professeur \\ Département de travail social \\ Université du Québec à Montréal
}

Un nombre croissant de familles sont appelées à prendre en charge une ou un proche adulte dépendant (un parent âgé en perte d'autonomie, un proche handicapé physique ou mental). Elles le seront de plus en plus en raison, d'une part, des politiques de désinstitutionnalisation proposées par l'État, et, d'autre part, de l'augmentation de la population adulte aux prises avec des dépendances de plus en plus sévères et chroniques. II n'est plus à démontrer que les familles et les femmes particulièrement constituent la pierre angulaire de l'organisation

1. Nous remercions les évaluatrices et évaluateurs anonymes d'une première version du présent article pour leurs critiques constructives qui nous ont permis de préciser certaines idées et de rendre l'article plus clair. 
des soins aux adultes dépendants et que le maintien à domicile ne pourrait se faire sans l'apport de celles-ci (Garant et Bolduc, 1990; ministère des Affaires sociales, 1985: 17 ; Thériault, 1991). Les institutions limitant pour la plupart leurs interventions aux malades en phase aiguë, c'est aux familles que revient la plus grande part du travail quotidien de la prise en charge.

Or, que veut dire s'occuper d'une ou d'un proche adulte dépendant à domicile?

Bon nombre de recherches et de pratiques en maintien à domicile traitent cette responsabilité de façon isolée des activités de la vie quotidienne des soignantes (dites aidantes naturelles) ${ }^{2}$ et limitent souvent leur vision de la prise en charge à un travail de soins directs à la personne dépendante. Tout en reconnaissant que les soins occupent une place majeure, cette vision demeure réductrice de la réalité.

Cette approche est remise en question dans le présent article, qui montre qu'au-delà des soins s'impose un important travail de conciliation visant à maintenir un équilibre entre les multiples besoins et exigences de chacune des sphères de la vie: sphères de la vie personnelle, sociale et familiale; sphères liées aux exigences du travail salarié et à la prise en charge. Ce travail s'ajoute aux deux autres types de travail, le travail de soins et le travail de mobilisation et de coordination des ressources, qui, selon nous, forment ensemble les composantes essentielles et indissociables du travail global de la prise en charge par les familles ${ }^{3}$.

Seule une lecture qui tient compte de l'interaction dynamique entre la responsabilité des soins et le contexte de la vie quotidienne de la soignante peut aider à saisir la nature de cette réalité. En ce sens, les perspectives théoriques proposées par l'analyse interactionnelle des soins (Corbin et Strauss, 1988 et 1990) et les analyses critiques de I'univers domestique (Cresson, 1991 ; Saillant, 1991; Vandelac et al., 1985) sont devenues centrales dans notre démarche en ce qu'elles nous ont permis de mieux cerner dans leur matérialité les différentes facettes de la prise en charge par les familles.

2. Nous avons préféré le terme "soignante » à celui d' "aidante naturelle», parce qu'il permet de briser l'image de la prise en charge comme étant une activité qui émane «naturellement» des individus, qui va de soi, ce qui n'est pas le cas. De plus, il s'agit d'un choix qui veut marquer de façon explicite que la prise en charge constitue un véritable travail de soins, reconnu comme tel dans le cadre institutionnel. Pour une critique de l'idéologie naturaliste, voir Guberman, Maheu et Maillé (1991) et Maheu et Guberman (1992).

3. Pour de plus amples détails sur le travail global de prise en charge, voir Maheu et Guberman, 1992. 
Les données de nos recherches menées depuis 1987 sur la prise en charge par les familles (Guberman, Maheu et Maillé, 1991; Guberman, Dorvil et Maheu, 1988), dont celle sur le travail et les soins (Guberman, Maheu et Maillé 1993) ${ }^{4}$, serviront de matériau de base pour illustrer notre lecture. Ces recherches visaient à comprendre, notamment, ce que signifie prendre soin d'un proche adulte dépendant dans le cadre de la famille et comment les soignantes arrivent à concilier les exigences du travail salarié, de la prise en charge et celles de leur vie personnelle et familiale.

De nature essentiellement qualitative, nos recherches ont privilégié une collecte de données basée sur des entrevues en profondeur de type semi-dirigé avec des proches, en majorité des femmes, assumant la prise en charge d'un des leurs, et sur l'utilisation du focus group avec des intervenantes et des intervenants actifs dans le champ du maintien à domicile ${ }^{5}$. L'analyse des données s'est largement inspirée de l'analyse de contenu classique (Bardin, 1977) et de la "grounded theory method» (Glaser et Strauss, 1967). Nos études incorporent tant des situations de prise en charge de personnes âgées en perte d'autonomie que de personnes aux prises avec des problèmes de santé physique ou mentale.

Après une brève présentation de quelques éléments de la problématique théorique de la prise en charge, nous examinerons comment le travail de conciliation s'actualise dans le quotidien des femmes qui sont à la fois soignantes et travailleuses salariées. Nous dégagerons, en conclusion, quelques implications pour la pratique en maintien à domicile.

\section{Problématique théorique}

Quelques études ont commencé à mettre en évidence la vision limitée du travail de prise en charge d'un proche adulte dépendant dans les familles qui traverse les pratiques et les politiques actuelles de maintien à domicile.

Les résultats préliminaires de notre recherche en cours (Guberman, Maheu et Maillé, 1994) portant sur ces pratiques et politiques révèlent

4. Ces recherches ont pu être menées grâce à des subventions obtenues respectivement de Subventions nationales au bien-être social de Santé et Bien-être social Canada, de la Commission d'enquête sur les services de santé et les services sociaux et du Conseil de recherche en sciences sociales.

5. Pour les fins de cet article, seuls les témoignages de quelques-unes des soignantes interviewées ont été retenus pour fonder nos propos. 
que les processus d'allocation de ressources et de soutien aux familles et à leurs proches relèvent $d^{\prime}$ 'une logique normative et médicale axée principalement sur les personnes dépendantes et les soins nécessaires à leur autonomie. Les particularités du contexte et des conditions des personnes assumant leur prise en charge ne font l'objet que d'une attention secondaire.

Ces observations sont corroborées par une étude récente du ministère de la Santé et des Services sociaux qui porte sur une évaluation de la prestation de services aux personnes âgées dans les CLSC et les centres hospitaliers (Trahan, Bélanger et Bolduc, 1993). Cette étude met en évidence deux problèmes. L'approche est principalement axée sur la pathologie: les évaluations sont incomplètes par rapport à la dimension psychosociale. Il y a absence d'une approche globale des problèmes vécus par les familles concernées et les conditions de cellesci ne sont pas prises en compte.

\section{QUELQUES ÉLÉMENTS DE CONTEXTE INCONTOURNABLES}

Notre démarche de recherche sur la prise en charge des proches adultes dépendants nous a révélé que ce travail ne peut être saisi dans sa globalité et sa complexité sans la prise en compte du contexte et des conditions particulières dans lesquels il s'actualise. À cet effet, les caractéristiques de la prise en charge à domicile et les transformations majeures qui ont traversé les familles et les politiques sociales ces dernières années, dont le travail salarié des femmes, le désengagement de l'État et l'alourdissement des populations dépendantes, constituent des éléments essentiels à la compréhension de cette réalité. La prise en compte de ces éléments de contexte nous apparaît incontournable en ce que ceux-ci définissent le caractère spécifique de la prise en charge dans les familles. Ils permettent de mieux saisir la réalité globale et invisible du travail requis et d'évaluer de façon plus juste ses implications pour les membres concernés dans le contexte des familles.

\section{Le travail salarié des femmes}

En raison de la place centrale qu'occupent les femmes dans l'univers des soins aux proches, il importe de tenir compte des récentes transformations dans leur situation socio-économique et plus particulièrement de leur entrée sur le marché du travail. En effet, la participation des femmes au travail salarié a plus que doublé au cours des quatre dernières décennies, passant de $25 \%$ en 1951 à $54 \%$ en 1990 . Plus de $70 \%$ des femmes qui ont entre 20 et 45 ans occupent un emploi. Non 
seulement les femmes entrent-elles de plus en plus sur le marché du travail, mais elles sont moins nombreuses à interrompre leurs activités (De Sève, 1988).

Si les femmes de plus de 55 ans qui constituent actuellement la majorité des soignantes auprès de proches malades ou dépendants ne sont pas nombreuses à occuper un emploi salarié, leurs cadettes ont, et auront de plus en plus, à faire face à la fois aux responsabilités découlant de leur situation de salariées et à celles de la prise en charge d'un proche adulte malade: parent âgé, conjoint malade, enfant adulte handicapé ou psychiatrisé. Des études évaluent qu'environ un tiers des soignantes actuelles sont aussi sur le marché du travail (Lesemann et Chaume, 1989; Stone et al., 1987). Mais ce chiffre s'élève à près de $50 \%$ lorsqu'on ne considère que les enfants-soignants sans tenir compte des conjoints et conjointes qui jouent aussi ce rôle (Stone et al., ibid.). Par ailleurs, une récente étude canadienne menée auprès de plus de 5000 travailleuses et travailleurs salariés (CARNET, 1993) révèle que $34 \%$ de ces personnes offrent de l'aide à un parent âgé, tandis que $12 \%$ participent aux soins personnels quotidiens, effectuant ainsi en moyenne neuf heures par semaine. De plus, $26 \%$ des employés assument des responsabilités à la fois auprès d'un parent âgé et de leurs enfants mineurs.

Ce premier élément soulève toute la question de la difficile sinon de l'impossible articulation du travail salarié au travail de la prise en charge et au travail domestique, de même que de tout le travail nécessaire pour réaliser celle-ci.

\section{L'alourdissement de la population adulte dépendante}

Ce ne sont plus uniquement des personnes en légère perte d'autonomie (personnes visées initialement par les programmes de maintien à domicile) que les familles sont appelées à prendre en charge, mais des personnes non autonomes. Le rapport Rochon en faisait le constat dès 1988 en affirmant que «les personnes maintenues à domicile sont aussi âgées et souvent aussi mal en point que celles qui se trouvent en centre d'accueil et avec beaucoup moins de services pour pallier à leur perte d'autonomie» (MSSS, 1988: 46).

Deux facteurs principaux ont contribué à ce changement: a) I'accroissement significatif des populations dépendantes aux prises avec des troubles sévères et chroniques en raison du vieillissement de la population et des développements de la pharmacologie et de la technologie médicale; b) le désengagement de l'État, la désinstitutionnalisation et la réduction de services institutionnels. 
Le travail des soins dans les familles s'est donc transformé. Nous assistons à un véritable transfert de soins institutionnels vers le domicile. Les familles sont non seulement appelées à prodiguer des soins spécialisés, mais à devoir également mobiliser et coordonner un ensemble de ressources professionnelles et de services externes pour assurer le maintien du proche dans le milieu.

\section{Les caractéristiques de la prise en charge dans le contexte des familles}

Contrairement à l'institution où toute l'organisation est axée sur les soins aux malades, la prise en charge dans le contexte des familles prend place à l'intérieur des activités de la vie quotidienne des personnes soignantes: leur vie personnelle, sociale et familiale et leur travail salarié. Elle s'ajoute et s'intègre à ces activités.

L'analyse critique de I'univers domestique de Vandelac et al. (1985) et l'analyse interactionnelle des soins de Corbin et Strauss (1988 et 1990) permettent de mettre en lumière le caractère propre que confère le contexte du domicile à la prise en charge. Ce faisant, elles font émerger tout le travail invisible qui entoure cette responsabilité.

Vandelac et al. (ibid.) avancent que l'univers du monde domestique obéit à une tout autre logique que celle du monde du travail. Ici, il y a enchevêtrement des différentes sphères de vie, qui n'existent pas en pièces détachées. II y a accomplissement de tâches simultanées, concomitantes et juxtaposées, qui ne sont pas hachurées, découpées comme dans le monde du travail. Corbin et Strauss (ibid.) appliquent cette logique aux soins prodigués par les familles et montrent que ces soins sont inextricablement liés aux différentes sphères de vie des membres concernés: sphères de la vie personnelle, sociale et familiale et sphère du travail salarié. II y a interdépendance et constante interaction. Un équilibre doit être maintenu entre les besoins du proche dépendant et ceux associés à chacune des sphères de vie. Les auteurs avancent que cette finalité occupe une place centrale dans le travail de prise en charge dans les familles et est assurée par ce qu'on appelle le travail de conciliation. Il s'agit d'un travail invisible fait d'acrobaties, de négociations, d'ajustements et de réajustements de toutes sortes.

Ces trois éléments de contexte déterminent et définissent le caractère particulier de la prise en charge dans les familles. Ils font que celle-ci va au-delà d'un travail de soins et englobe un important travail de mobilisation de ressources, de coordination et de conciliation entre de multiples besoins souvent conflictuels. La logique actuelle des services n'arrive pas à rendre compte de ce travail invisible et, ce faisant, renvoie une image réductrice de cette réalité. 
Dans les pages qui suivent, nous examinerons une des facettes de la prise en charge dans les familles, le travail de conciliation.

\section{LE TRAVAIL DE CONCILIATION}

Le travail de conciliation vise à mettre en place un ensemble de formes d'aménagement afin d'assurer le maintien d'un équilibre entre les besoins du proche dépendant et les exigences associées aux différentes sphères de vie des personnes soignantes.

Ces formes d'aménagement constituent un ensemble de moyens ou de stratégies auxquels ont recours les personnes soignantes dans chacune de leurs sphères de vie.

Dans la sphère du travail salarié, ces formes renvoient à un réaménagement des conditions de travail, à la recherche d'un type d'emploi compatible avec les exigences de la prise en charge ou au retrait partiel ou total du marché du travail pour une période prolongée. Dans la sphère de la vie familiale, les soignantes ont recours à des moyens tels que le partage des tâches liées à la vie familiale, la réorganisation du travail domestique, la réduction et l'élimination des aspects non essentiels de la vie familiale. Quant à la sphère de la vie personnelle et sociale, les soignantes I'aménagent en empiétant sur leurs soirées ou leurs congés, en réduisant ou annulant le temps consacré à leurs activités personnelles et sociales, en modifiant leurs habitudes de vie et en changeant leur façon d'être. Finalement, dans la sphère de la prise en charge, les soignantes font appel à deux stratégies: le recours au soutien de la famille, des services publics, privés et communautaires et la mise en place de mécanismes de contrôle ou de réduction du travail de prise en charge.

Ce rapide coup d'œil sur les formes d'aménagement révèle en soi un important travail de gestion, d'organisation et de coordination de tout un système. La personne dépendante et les soins à lui prodiguer ne constituent donc qu'un élément qui se trouve en interaction constante avec chacune des sphères de vie de la personne soignante. Ce travail de gestion sous-tend toute une habileté en ce qui a trait aux décisions à prendre, aux «choix » à faire quant aux changements, aux ajustements à effectuer, à l'établissement des priorités et à l'évaluation des conséquences du choix des formes d'aménagement en vue de maintenir un certain équilibre entre les sollicitations et les exigences des diverses sphères. Les "choix» en question (qui ne relèvent pas toujours de l'ordre des décisions rationnelles) sont conditionnés par plusieurs facteurs, dont la situation socio-économique et familiale des soignantes, la nature et les conditions du travail salarié, le degré d'autonomie de 
la personne dépendante ainsi que ses attentes, l'accès au soutien, les valeurs et l'image de soi des soignantes.

L'examen de la situation de quelques-unes des soignantes interviewées permettra d'illustrer comment ce travail de conciliation s'actualise dans la réalité, et ce à l'intérieur de chacune des sphères de la vie quotidienne. Il met aussi en relief les efforts de planification, de négociation et de coordination requis; enfin, il nous permet de comprendre comment les exigences de chacune des sphères de vie sont interreliées, interactives et satisfaites grâce au maintien entre elles d'un équilibre, qui néanmoins demeure toujours fragile en raison des fluctuations et de l'évolution des événements.

\section{Sphère du travail}

Une des tâches les plus fréquemment accomplies par les soignantes et qui a un impact sur leur travail salarié est l'accompagnement de la personne prise en charge aux rendez-vous médicaux. Il est à noter que ces rendez-vous sont presque toujours fixés durant les heures du travail. Or, le simple fait d'avoir à se libérer de son travail salarié pour quelques heures peut impliquer toute une organisation, comme nous I'explique Stéphanie ${ }^{6}$, infirmière à temps plein qui s'occupe de sa mère en perte d'autonomie.

C'est sûr que ça peut me demander des changements d'horaire. $\mathrm{Si}$ je le demande assez à l'avance à la personne qui fait les horaires, ça s'arrange toujours. Quand je connais la date des rendez-vous 2 ou 3 mois d'avance, je lui dis «Donne-moi telle journée de congé, parce que j'ai un rendez-vous pour maman ». Mais la semaine prochaine, par exemple, maman a un rendezvous qui ne tombe pas sur ma journée de congé. Eh bien, c'est moi qui dois surveiller les horaires de tout le monde, puis je finis par demander à une fille si elle peut changer de jour avec moi et on s'entend là-dessus.

L'organisation d'un rendez-vous devient un travail impliquant des négociations avec des supérieurs pour la libération du temps de travail, avec des collègues pour l'aménagement du travail et avec les professionnels de la santé pour l'obtention et l'entente de la date du rendez-vous. Ce travail dépend d'un ensemble de facteurs qui vont de la bonne volonté et la compréhension des employeurs et des pairs aux conditions de travail et aux contraintes liées aux services. Ainsi, la

6. Les noms des soignantes ainsi que certains détails de leur situation ont été modifiés afin de protéger leur anonymat. 
réorganisation de son horaire n'est pas sans conséquences pour Stéphanie:

Des fois je suis obligée de travailler avec le quart de soir et de rentrer travailler le lendemain matin à $8 \mathrm{~h}$, justement pour avoir une journée de congé pour maman. Sauf que c'est moi qui le paie $d^{\prime}$ une certaine façon, parce que parfois je suis obligée de faire des horaires un peu fous, pour arriver à faire ça.

Ce qui apparaît être une simple tâche d'accompagnement à un rendez-vous médical implique tout un travail de négociation, de coordination, de mobilisation de ressources qui peut aller jusqu'à affecter la santé physique et mentale de Stéphanie à cause du manque de sommeil.

Une autre forme d'aménagement extrêmement importante pour un grand nombre de mères et de soignantes salariées est le recours au téléphone pour vérifier la situation à la maison ou pour organiser des rendez-vous, prendre des informations auprès des professionnels (CINBIOSE, 1993 ; Guberman et al., 1993). Or, I'utilisation du téléphone n'est pas toujours permise dans les milieux de travail.

Bernadette, préposée aux soins dans un hôpital, mère d'un fils paraplégique et $d^{\prime}$ un autre diagnostiqué maniaco-dépressif, a même dû changer de poste afin d'avoir accès à un téléphone lui permettant $\mathrm{d}^{\prime}$ assurer la surveillance à distance de ses fils.

Je me suis arrangée pour pouvoir utiliser le téléphone durant mes heures de travail. Parce qu'en fin de compte, en étant à I'entretien, si je laisse mon chariot durant vingt minutes pour faire un appel, ça dérange personne. Tandis qu'avant quand j'étais au nursing... ça demande tellement auprès des patients, tu peux pas laisser un patient... S'ils te demandent d'aller porter un gaz artériel, ou qu'il y a un patient qui est en train de t'appeler, tu peux pas prendre vingt minutes pour aller téléphoner.

\section{Sphère de la vie familiale}

Pour certaines soignantes, la conciliation passe par l'organisation rigide de chaque moment de la journée. En répétant de jour en jour les mêmes activités à la même heure, elles réduisent les problèmes et le temps requis pour la planification, ce qui transforme la conciliation en une forme de routine.

Carmen est mariée à un homme atteint d'une maladie dégénérative qui l'empêche de travailler. Le couple a une fille de huit ans. Carmen s'occupe aussi de sa belle-mère qui habite avec eux. Cette 
dernière souffre d'emphysème, d'asthme, de diabète, en plus d'une maladie coronarienne, et elle a besoin de surveillance constante, même si elle est relativement autonome dans ses activités de la vie quotidienne. Carmen travaille à temps plein.

Je suis debout à 5 heures et demie le matin. À 7 heures moins quart je pars de la maison. À 8 heures ma petite s'en va, ma belle-mère se lève, elle fait son lit, puis elle fait le lit de la petite. Après, elle va s'étendre jusqu'à 11 heures parce qu'elle est trop faible. À midi moins quart la petite arrive et ma belle-mère lui prépare son dîner. Mon mari est là pour surveiller le tout. II y a une de ses soeurs qui vient voir sa mère à tous les jours de 2 heures jusqu'à 4 heures moins quart, ce qui permet à mon mari de sortir prendre une marche ou de faire des commissions. J'arrive à la maison vers 4 heures, je commence à préparer le souper; c'est des choses vite faites. De 6 heures jusqu'à 7 heures, $c^{\prime}$ est les devoirs et les leçons de ma fille. De 7 à 8 , eh bien je monte, puis je regarde la télé. À 8 heures je suis couchée, 8 heures, 8 heures et demie.

L'examen de cette organisation hyper-structurée montre que Carmen a dû faire appel à plusieurs acteurs en vue de s'assurer de la participation de chacun au travail domestique et à la prise en charge. C'est sans doute grâce à un travail important de planification et de négociation qu'elle arrive à maintenir un certain équilibre lui permettant de concilier travail, prise en charge et vie familiale. Mais à quel prix? $C^{\prime}$ est ce que se demandent les auteures d'une recherche sur la conciliation: "Comment ne pas trouver absurde de vivre ainsi sa vie, à l'avance, en la planifiant sans la vivre, au point d'en perdre le goût de vivre? » (CINBIOSE, 1993: 38).

La situation de Lorraine, mariée, travailleuse à temps plein et mère de cinq enfants dont un fils de 19 ans atteint de paralysie cérébrale et d'autisme, illustre on ne peut mieux l'ampleur des efforts que doivent déployer les soignantes pour assurer le partage des activités domestiques afin de s'occuper de la personne prise en charge ou de s'accorder quelques moments de répit.

On avait tout mis sur une liste, une belle liste sur ordinateur: sortir les déchets 2 fois par semaine (disons que ça prend 10 minutes à chaque fois), raconter une histoire à Xavier le soir (15 minutes), descendre le panier de lavage... tout, tout était minuté. En tout cas, j'avais tout décortiqué (...), j'avais à peu près 75 heures de tâches ménagères. Ça faisait trois, quatre dimanches matin qu'on faisait des dîners-causerie pour en arriver à mettre sur papier ce qu'on faisait. Puis, le jour où tout été sur ordinateur et qu'on l'a eu devant nous, mon mari a pris sa tasse de café, puis est parti en disant qu'il s'occupait de payer 
les comptes. Alors, mon fils aîné a dit: «Tu vois, papa il en a quasiment pas de tâches, pourquoi j'en prendrais, moi ? ॥ II est parti. Puis on est restées, Ève et moi, et on a dit: «Bon, qu'est-ce qu'on fait avec ça?»

II arrive parfois que la «lutte» à mener pour parvenir au partage des tâches requiert tellement de temps et d'énergie que les soignantes estiment plus facile en matière de conciliation de tout faire ellesmêmes.

\section{Sphère de la prise en charge}

Même dans la sphère de la prise en charge, le travail à accomplir ne se limite pas aux soins directs à prodiguer à la personne malade. En effet, pour arriver à offrir ces soins, les soignantes doivent tout d'abord procéder à une évaluation des alternatives, ainsi que des besoins de la personne à soigner, combiner une variété de ressources et les ajuster aux besoins. Parallèlement, il leur faut tenir compte de leur budget, des ressources dont elles peuvent disposer, de leurs valeurs ainsi que de celles des autres acteurs. En dernier lieu, il leur faut établir des priorités, trouver un compromis entre les besoins souvent opposés de la personne dépendante et ceux des autres membres de la famille, chercher à établir une certaine routine et fournir elles-mêmes les services non disponibles ailleurs (Balbo, 1987). Comme la prise en charge ne se fait pas de façon isolée, ces choix doivent tenir compte de la réalité des autres sphères. Le recours aux services externes introduit encore $d^{\prime}$ autres acteurs et d'autres exigences à considérer.

Colette, avocate dont la mère est en grande perte $d^{\prime}$ autonomie, a placé l'embauche de personnel infirmier au centre de ses formes d'aménagement. Mais, au-delà du travail pour trouver les personnes nécessaires, cette forme de conciliation, visant à permettre à Colette de maintenir son emploi tout en assurant les soins nécessaires à sa mère, l'a obligée à transiger avec ses frères et sœurs qui lui déléguaient la tâche, en plus de devoir tenir compte des opinions de sa mère.

Pour moi, trouver de l'aide pour une personne âgée c'est encore plus difficile que pour un enfant. Parce qu'un enfant, quand je lui trouve une gardienne il va l'accepter, même s'il n'est pas content. Tandis que quand maman n'aimait pas sa gardienne, elle me disait tout simplement: "Celle-là je ne l'aime pas!" Alors je trouvais ça très difficile au point de vue émotif. Il fallait constamment que j'arrive à faire face à cette situation. D'un côté, il fallait qu'il y ait quelqu'un avec maman et de l'autre tout le monde me disait: "C'est toi qui connais les gens dans les hôpitaux, c'est toi qui connais ce milieu-là, trouve du monde!» 
Monique nous parle de l'important travail de coordination des professionnelles exigé par le placement de son père.

Il a fallu que je négocie d'une certaine façon; il fallait régler les affaires au niveau de l'hébergement de papa, et aussi au niveau de son suivi médical, etc. Alors je me suis mise à faire un gros travail auprès de la travailleuse sociale et du médecin et du personnel hospitalier. J'ai contraint les gens à s'occuper de leurs affaires, puis à bien le faire. J'ai dit: "Je veux qu'on ait des rencontres, des rencontres d'évaluation. On va établir les raisons pour lesquelles le placement n'a pas fonctionné, puis on va essayer de voir ce qu'il faudrait corriger à I'avenir, et sur quoi il faut miser, etc. » J'ai dit: "On va essayer d'établir un plan d'intervention. » En faisant ça, ç'a fonctionné, ce qui fait que les infirmières aussi, je les mettais dans le coup. Finalement, en établissant une certaine complicité, moi je me suis libérée, parce que tout le monde a fait sa part, au moment où il le fallait, et ç'a été beaucoup plus facile. Alors, en mettant tout ça en commun, il me semble que là, ç'a simplifié les choses, (...) ç'a été un long travail, un long travail, mais qui a été profitable.

On est donc loin des soins directs à procurer à une personne dépendante. Dans plusieurs cas, la prise en charge consiste principalement dans la mobilisation et la coordination des ressources humaines et matérielles essentielles à la conciliation. Les problèmes liés, par exemple, au recours à des gardiennes ou à du personnel de maintien à domicile obligent les soignantes à effectuer de savants calculs. Elles doivent en effet évaluer la pertinence d'investir du temps et de l'énergie pour trouver les ressources appropriées, suffisantes et disponibles au bon moment, plutôt que de réduire leur présence dans d'autres sphères en prenant des congés sans solde ou bien en éliminant leurs activités sociales. Lorraine nous en parle:

La semaine prochaine, j'ai le choix d'aller à un congrès pendant deux jours ou de faire un aller-retour le même jour parce que ça simplifie la tâche de gardiennage. (...) Moi ça me tente drôlement de rester les deux jours, sauf que je ne vois pas comment je vais $\mathrm{m}^{\prime}$ organiser. Il faut que je passe beaucoup de temps à essayer de régler ça. Une fois, par exemple, j'ai fait des photocopies d'un plan de toutes les fois où j'avais besoin de gardiennes pendant plusieurs mois, puis j'ai passé le plan aux monitrices des loisirs, je l'ai diffusé un peu. Mais personne n'était disponible pour aucune des fois où j'en avais besoin. 


\section{Sphère de la vie personnelle et sociale}

En plus de mettre en place des arrangements pour assurer, pendant le jour, la prise en charge de la personne dépendante selon ses besoins, il faut aussi voir à ce que le travail domestique et le travail de maternage, du moins pour l'essentiel, soient accomplis et que les exigences au travail soient remplies. Les soignantes doivent donc jongler avec plusieurs scénarios quant à l'organisation de leur temps.

Lorraine nous explique aussi comment à tout moment il faut mesurer la portée de chaque décision relative à l'utilisation de son temps.

Le matin, je mets une demi-heure à préparer mon fils à partir. Cette demi-heure-là, c'est sûr que je la dormirais ou bien je ferais autre chose. Je $\mathrm{m}^{\prime}$ habillerais tranquillement, puis je serais moins pressée de partir. Mais il faut que je prenne le temps nécessaire pour lui pour qu'il ne parte pas sur un mauvais pied, parce que je sais que sa journée va s'en ressentir.

\section{LE TRAVAIL DE CONCILIATION: UN ÉQUILIBRE FRAGILE}

L'équilibre que permet d'atteindre le travail de conciliation des exigences des diverses sphères de la vie courante et des soins au proche n'est jamais assuré et mis en place de façon permanente et stable. En ce sens, il s'agit toujours d'un équilibre relatif (Corbin et Strauss, 1988: 98), un équilibre fragile. Tenter de tout concilier s'apparente le plus souvent à marcher sur une corde raide (Charmaz, dans Corbin et Strauss, 1988 : 98).

Certaines formes $d^{\prime}$ 'aménagement peuvent être relativement stables et d'autres temporaires selon les fluctuations et l'évolution des événements (Corbin et Strauss, 1990:64-65). Mais, en général, rien n'est jamais acquis. Bon nombre des aménagements sont sujets à renégociation et à réarticulation en raison de déterminants tels que la forte interdépendance entre la prise en charge et les autres sphères de la vie quotidienne de la soignante, d'une part, et les changements des situations et des conditions dans le temps, d'autre part (contingences de la vie quotidienne, imprévus, crises, détérioration de l'état de santé du proche dépendant, perte d'emploi du conjoint, maladie d'un autre membre de la famille, changement dans les conditions de travail, banque de congés épuisée, coupure ou diminution des ressources, etc.). Tout changement dans l'une ou l'autre sphère affecte le fragile équilibre si difficilement mis en place. 
Le travail de conciliation se résume souvent à une vaine tentative de vouloir rendre stables des situations qui sont par nature instables.

Colette pensait être en mesure de poursuivre son travail comme avocate en engageant du personnel infirmier pour sa mère. Mais, comme elle l'explique, il y a toujours des imprévus qui exigent des changements et des révisions de la situation.

Finalement, on avait réussi à trouver du personnel 24 heures sur 24. Mais, ça ne finit pas là. Souvent, j'étais au bureau et une des personnes appelait et me disait: "Écoute, je ne peux pas être là demain .» J'essayais de trouver une autre personne, sinon, souvent c'est moi qui manquais le bureau. Finalement, l'année dernière, (...) deux membres du personnel, qui étaient réguliers, nous ont avertis qu'ils ne pouvaient plus s'en occuper. $C^{\prime}$ est à ce moment-là qu'on a dû réviser notre façon d'agir, parce qu'on ne pouvait lui imposer des visages nouveaux, elle ne les voulait plus, les visages nouveaux.

Claire, mère d'une fille de 26 ans, épileptique et affligée de multiples handicaps, travaille à temps plein comme cuisinière-chef. Pour accompagner sa fille aux rendez-vous médicaux, elle avait l'habitude de s'absenter du travail pour quelques heures. Mais comme elle l'explique, la situation au travail a changé.

Dans le temps j'avais deux employés. Maintenant ils ont "coupé », j'ai juste un employé. Avant je partais du travail à $10 \mathrm{~h}$ et demie après la pause. J'arrivais ici à $11 \mathrm{~h}$ moins quart, puis à $11 \mathrm{~h}$ j'étais rendue avec ma fille chez le neurologue. Elle nous voyait tout de suite. Je repartais tout de suite et je ramenais Patricia à la maison pour que la gardienne la reprenne. Puis je continuais au travail. Mais ça, je ne pourrai plus le faire, ça m'enlève une porte de sortie. Quand j'avais deux employés, ils pouvaient se débrouiller, j'étais moins inquiète...

Claire a toujours compté sur les services d'une école spécialisée et sur les services de loisirs pour l'été. Or, cette année, la situation a changé, l'obligeant à renégocier ces formes d'aménagement.

L'été, Patricia allait aux loisirs communautaires depuis 3 ans. Cette année, ils l'ont refusée parce qu'elle n'est pas assez autonome. C'est parce que le gouvernement a coupé dans les subventions que le terrain de jeu recevait. (...) Pour avoir soin de 46 handicapés, il y avait seulement 5 moniteurs. Ils n'avaient donc plus la disponibilité pour Patricia. J'ai commencé à chercher ailleurs et Patricia aurait eu droit à un service, un camp de I'OPHQ, mais il faut que son travailleur social signe un papier. Or, son travailleur social n'a jamais voulu signer ce fameux papier parce qu'il voulait placer ma fille dans une 
famille d'accueil pour qu'elle devienne autonome. J'ai même appelé le directeur général du CLSC.

\section{Les formes d'aménagement: choix ou contraintes}

À I'instar des auteurs du rapport de recherche de CINBIOSE, il faut reconnaître que ces formes d'aménagement constituent « un art consommé de l'efficacité et de la débrouillardise et que les interviewées nous ont impressionnés à plus d'une reprise» (1993: 36). Mais elles nous apparaissent comme relevant plus de contraintes que de choix rationnels. "Ce slalom incessant, cette course folle contre la montre, stressante, épuisante, aux revers pervers innombrables... et aux coûts personnels et sociaux exorbitants est loin de constituer un idéal de vie» (ibid.).

Tout en reconnaissant que les personnes soignantes sont des "acteurs actifs» dans leurs choix et leurs actions, dotées d'une rationalité propre et non victimes de leurs conditions et de leurs contraintes, nous croyons que le risque est grand de tomber dans le piège de «l'acteur souverain et rationnel, un modèle profondément ancré dans notre culture» (Crozier et Friedberg, 1977: 45 ) qui évacue les conditions dans lesquelles I'acteur fait ses choix et met en œuvre ses actions. Ces auteurs rappellent deux choses: a) «que l'acteur n'a que rarement des objectifs clairs et cohérents»; b) qu'il est illusoire et faux de considérer son comportement comme toujours réfléchi, $c^{\prime}$ est-à-dire médiatisé par un sujet lucide, calculant ses mouvements en fonction d'objectifs fixés au départ; c) qu'il est essentiel de «relier la conduite de l'acteur au contexte dans lequel il opère, découvrir les conditions matérielles qui limitent et définissent ses choix et partant le sens de ses comportements» (ibid.: 47).

La conciliation et le maintien d'un équilibre ne sont pas uniquement une affaire de bonnes ou mauvaises formes d'aménagement ou stratégies ou l'art de bien s'organiser ou de faire des choix justes. Une telle conception réduit les problèmes de conciliation à ceux des personnes et est dangereusement myope. Ces problèmes résultent pour I'essentiel de l'augmentation des contraintes professionnelles et familiales, de l'inadéquation des conditions de travail et de la pénurie de services collectifs (CINBIOSE, 1993: 9).

\section{CONCLUSION}

Le présent article a tenté de démontrer qu'en raison des éléments de contexte propres à la prise en charge par les familles, celle-ci ne peut 
se réduire à un travail de soins, mais englobe, notamment, un important travail de conciliation pour maintenir un équilibre entre les exigences de toutes les sphères de la vie et celles de la prise en charge. L'épuisement des soignantes ou leurs absences répétées à leur emploi rémunéré ne sont plus alors seulement l'expression d'une surcharge due aux soins requis par le ou la proche, mais aussi l'expression d'une impossibilité à maintenir stable ce qui en soi est instable et à concilier ce qui est souvent inconciliable. Seule une lecture qui intègre les propriétés du contexte de la prise en charge et l'interaction entre la maladie, les soins et les activités de la vie quotidienne des soignantes permet une compréhension représentative de cette réalité et de sa portée pour les familles et particulièrement pour les femmes dans celles-ci.

Une telle lecture n'est pas sans avoir des implications pour la pratique en maintien à domicile.

Dans leur pratique d'évaluation des besoins et d'allocation des ressources, les intervenants et intervenantes doivent mettre au centre de leur démarche non seulement les soins requis par la personne dépendante, mais le contexte dans lequel s'insèrent ceux-ci et les conditions des personnes soignantes concernées. Le travail salarié des soignantes apparaît comme un des éléments de contexte auxquels on doit porter une attention particulière.

Par ailleurs, dans un contexte de pratique où les problèmes sont codés et prédéfinis, il est impérieux que les intervenants et les intervenantes se rapprochent du terrain et se fassent plus inductifs, afin de mieux saisir la complexité et les multiples dimensions des réalités sociales sur lesquelles ils sont appelés à intervenir. Ils doivent faire en sorte de rendre visible et de mettre en évidence la riche information que leur révèle leur expertise sociale en ce qui regarde ces réalités et leur complexité. Cela pourrait constituer un point de départ pour une remise en question de la logique médicale et normative qui prévaut actuellement. 


\section{Références bibliographiques}

BALBO, L. (1987). "Crazy Quilts: Rethinking the Welfare State Debate from a Woman's Point of View», dans A.S. Sassoon (dir.), Women and the State. Londres: Unwin Hyman, p. 45-71.

BARDIN, L. (1977). L'analyse de contenu. Paris: PUF.

CARNET (1993). Work and Eldercare Research Group, Work and Family: The Survey. Guelph : The Canadian Aging Research Network.

CINBIOSE (1993). Concilier l'inconciliable, étude exploratoire sur la conciliation des responsabilités familiales et professionnelles dans trois milieux de travail. Montréal : UQAM.

CORBIN, J. et A. STRAUSS (1988). Unending Work and Care, Managing Chronic IIIness at Home. San Francisco: Jossey-Bass Inc.

CORBIN, J. et A. Strauss (1990). "Making Arrangements: The Key to Home Care », dans J. GUBRIUM et A. SANKAR (dir.), The Home Care Experience. Newbury Park: Sage.

CRESSON, G. (1991). "La santé, production invisible des femmes », Recherches féministes, vol. 4, $\mathrm{n}^{\circ} 1: 31-44$.

Crozier, M. et E. FriedberG (1977). L'acteur et le système. Paris : Éditions du Seuil.

DE SEVE, M. (1988). "Pour une mise à jour des caractéristiques de I'emploi féminin de 1961 à 1986 », Interventions économiques, nº 20-21: 59-101.

GARANT, L. et M. BOLDUC (1990). L'aide par les proches: mythes et réalités. Études et analyse, no 8, Québec: MSSS, Direction de l'évaluation.

Glaser, B. et A. StRAuSS (1967). The Discovery of Grounded Theory. Chicago: Aldine.

Glaser, N. (1990) «The Home as Workshop: Women as Amateur Nurses and Medical Care Providers », Gender and Society, vol. 4, n 4 : 479-499.

Guberman, N., P. MAHEU et C. MAILlÉ (1994). Femmes soignantes, travail salarié et politiques sociales de maintien en milieu naturel des personnes dépendantes. Recherche en cours financée par le CRSH.

Guberman, N., P. MAHeU et C. MAILlÉ (1993). Travail et soins. Montréal: Les Éditions du Remue-Ménage.

Guberman, N., P. MAHEU et C. MAILlé (1991). Et si l'amour ne suffisait pas. Familles, femmes, adultes dépendants. Montréal : Les Éditions du RemueMénage.

GuBERMAN, N., P. MAHEU et H. DORVIL (1987). Amour, bain et comprimé ou I'ABC de la désinstitutionnalisation. Rapport de recherche produit pour la Commission d'enquête sur les services de santé et les services sociaux. Québec: Les Publications du Québec.

LeSEMANN, F. et C. Chaume (1989). Familles-providence: La part de l'État. Montréal : Les Éditions Saint-Martin.

MAHEU, P. et N. GUBERMAN (1992). «Familles, personnes adultes dépendantes et aide "naturelle" : entre le mythe et la réalité ", Revue internationale d'action communautaire, 28/68: 51-62.

MINISTÈRE DES AFFAIRES SOCIALES (1985). Un nouvel âge à partager. Québec: Les Publications du Québec. 
Ministère de la SANTÉ eT DeS SeRviCes SOCIAuX (1989). Rapport de la Commission d'enquête sur les services de santé et les services sociaux. Québec : Les Publications du Québec.

NEAL, M.B. et al. (1993). Balancing Work and Caregiving for Children, Adults and Elders. Family Caregiver Applications Series, vol. 3, Newbury Park: Sage.

SAILLANT, F. (1991). "Les soins en péril: entre la nécessité et l'exclusion », Recherches féministes, vol. 4, $\mathrm{n}^{\circ} 1: 11-29$.

StONE, R. et al. (1987). "Caregivers of the Frail Elderly: A National Profile», The Gerontologist, vol. 27, n 5 : 616-626.

Strauss, A. (1987). Qualitative Analysis for Social Scientists. New York: Cambridge Press.

THÉRIAULT, V. (1991). Le maintien à domicile, Beaucoup de besoins, peu de ressources. Conseil de la santé et des services sociaux de Lanaudière et des Laurentides, CLSC des régions de Lanaudière et des Laurentides.

TRAHAN, L., L. BÉLANGER et M. BOLDUC (1993). Une évaluation de la prestation de services dans les CLSC et les centres hospitaliers. Québec: ministère de la Santé et des Services sociaux.

VANDELAC, L. et al. (1985). Du travail et de l'amour. Montréal : Les Éditions Saint-Martin. 